\title{
IL10 rs1800896 polymorphism is associated with liver cirrhosis and chronic hepatitis $B$
}

\author{
L.N. Cao' ${ }^{1}$, S.L. Cheng ${ }^{2}$ and W. Liu ${ }^{3}$ \\ ${ }^{1}$ Kidney Disease Department of Internal Medicine, Xianyang Central Hospital, \\ Xianyan, China \\ ${ }^{2}$ Department of General Surgery, Xianyang Central Hospital, Xianyan, China \\ ${ }^{3}$ Department of Thoracic Surgery, Xianyang Central Hospital, Xianyan, China \\ Corresponding author: W. Liu \\ E-mail: liuwen_liw@163.com \\ Genet. Mol. Res. 15 (1): gmr.15017256 \\ Received July 21, 2015 \\ Accepted November 4, 2015 \\ Published February 19, 2016 \\ DOI http://dx.doi.org/10.4238/gmr.15017256
}

ABSTRACT. We conducted a case-control study to assess the role of two IL10 gene polymorphisms ( $\mathrm{rs} 1800896$ and rs1800872) in susceptibility to liver cirrhosis, and their association with chronic hepatitis B in a Chinese population. A case-control study was designed to investigate the association between functional polymorphisms of IL10 (rs1800896 and rs 1800872) and the development of liver cirrhosis. Between March 2012 and March 2014, we recruited 241 patients with liver cirrhosis and 254 controls from Xianyang Central Hospital. Genotyping of IL10 rs1800896 and rs1800872 polymorphisms was carried out using the polymerase chain reaction coupled with restriction fragment length polymorphism. Using multivariate logistic regression analysis, we found that individuals with the AA genotype of IL10 rs1800896 showed an increased risk of liver cirrhosis compared with those with the GG genotype in a codominant model (OR = $2.01,95 \% \mathrm{Cl}=1.10-3.65)$. In dominant and recessive models, we found that the IL10 rs 1800896 polymorphism was correlated with the development of liver cirrhosis (for the dominant model, $\mathrm{OR}=1.46,95 \% \mathrm{Cl}=1.01-2.13$; for the recessive model, $\mathrm{OR}=1.72,95 \% \mathrm{Cl}=1.01-3.02)$. In summary, our 
study suggests that the IL10 rs1800896 polymorphism is associated with the development of liver cirrhosis.

Key words: Interleukin-10; Polymorphism; Liver cirrhosis; Chronic hepatitis B

\section{INTRODUCTION}

Hepatitis B virus-related liver diseases are severe health problem worldwide (Hsiang et al., 2015). Long-term chronic hepatitis $B$ virus infection may cause liver injury and promote progression to fibrosis and ultimately cirrhosis (Ansari et al., 2015). The development of chronic hepatitis is highly individual, and not all hepatitis B infection patients suffer from liver cirrhosis, which suggests that gene polymorphisms, environmental factors, and the characteristics of individuals influence the susceptibility to liver cirrhosis (Lin et al., 2013; Ansari et al., 2015).

Interleukin-10 (IL-10) is one of the most important immunoregulatory cytokines, and the gene that encodes it plays a fundamental role in anti-inflammatory and immunosuppressive activities, as well as downregulating type-1 T helper cell (Th1) cytokines. The Th1 cytokines are associated with increased necroinflammatory activity and histological fibrosis in chronic hepatitis B or C patients (Falasca et al., 2006). The authors of a previous experimental study reported that IL10 is associated with hepatic fibrogenesis, and patients with liver cirrhosis presented reduced liver inflammation and fibrosis when they received recombinant IL-10 therapy (Tsukamoto, 1998; Nelson et al., 2000). Therefore, the IL10 gene may be an important factor in the development of liver cirrhosis. We conducted a case-control study to assess the role of IL10 rs1800896 and rs1800872 polymorphisms in susceptibility to liver cirrhosis, and their association with chronic hepatitis $B$ in a Chinese population.

\section{MATERIAL AND METHODS}

\section{Patients}

A case-control study was designed to investigate the association between functional polymorphisms of IL10 (rs1800896 and rs1800872) and the development of liver cirrhosis. Between March 2012 and March 2014, we recruited 262 patients with liver cirrhosis from Xianyang Central Hospital. Liver cirrhosis was diagnosed by histopathological investigation, ultrasound, computed tomography, or magnetic resonance imaging. Patients who were positive for hepatitis $\mathrm{C}$ antibody and who had other liver diseases were excluded from our study. Ultimately, 241 patients agreed to participate in our study (participation rate $=91.98 \%$ ).

Healthy adult subjects without liver cirrhosis (297) were randomly recruited from individuals who came to receive regular health check-ups in our hospital between March 2012 and March 2014. Ultimately, 254 subjects agreed to participate in our study, with a participation rate of $85.52 \%$.

The demographic and clinical characteristics of the patients with cirrhosis and of the controls were collected using an ad hoc questionnaire and medical records, including information on gender, age, chronic hepatitis B infection, alcohol consumption, and Child-Pugh score. The alcohol consumption category was divided into non-drinkers and drinkers. The definition of a drinker was an individual who drank $>60 \mathrm{~g} /$ day of alcohol for more than 6 months. All liver cirrhosis 
patients and all controls signed written informed consent before enrolling in our study. The protocol of this study was approved by the Ethics Committee of the Children's Hospital of Zhengzhou.

\section{Genotyping}

Approximately $5-\mathrm{mL}$ peripheral blood samples were drawn from the liver cirrhosis patients and the controls, and the blood samples were stored at $-80^{\circ} \mathrm{C}$ until required. Genomic DNA was extracted from the peripheral blood using a TIANamp Blood DNA Kit (Tiangen Biotech, Beijing, China).

After extraction, genotyping of IL 10 rs 1800896 and rs 1800872 polymorphisms was carried out by polymerase chain reaction coupled with restriction fragment length polymorphism. Primer sequences for IL10 rs1800896 and rs1800872 were designed using the Sequenom Assay Design 3.1 software (Sequenom, San Diego, CA, USA). The forward and reverse primers for IL10 rs 1800896 were 5'-AGGATGTGTTCCAGGCTCCT-3' and 5'-CCCTTGTACAGGTGATGTAACA-3', respectively; the forward and reverse primers for rs 1800872 were 5 '-GGTGAGCACTACCTGACTA GC-3' and 5'-CCTAGGTCACAGTGACGTGG-3', respectively. The restriction enzymes for IL10 rs 1800896 and rs 1800872 were BseRI and Rsal, respectively. The digested fragments of the IL10 rs $1800896 \mathrm{G}$ allele were 33 and $106 \mathrm{bp}$, and those of the A allele were 33, 106, and $139 \mathrm{bp}$. The digested fragments of the rs 1800872 allele were 176 and $236 \mathrm{bp}$, and those of the $C$ allele were 176,236 , and $412 \mathrm{bp}$. Additionally, approximately $10 \%$ of the samples were randomly selected and retested, and the results were $100 \%$ concordant.

\section{Statistical analysis}

The statistical differences in the demographic and clinical characteristics between cases and controls were subjected to the chi-squared test. The distribution of genotypes of IL $10 \mathrm{rs} 1800896$ and rs 1800872 in the controls was tested for deviation from the Hardy-Weinberg equilibrium (HWE). We used the chi-squared test to examine differences in genotypic and allelic distribution between liver cirrhosis patients and controls. The odds ratios (ORs) and $95 \%$ confidence intervals (Cls) were evaluated using logistic regression models adjusted for confounding factors. Stratified analysis was conducted to analyze interaction between chronic hepatitis $B$ infection and genetic polymorphisms of $I L 10$ rs 1800896 and rs 1800872 in the risk of liver cirrhosis. A P value $<0.05$ was considered to indicate a statistically significant difference. All statistics were analyzed using the SPSS statistical package software, version 16.0 (SPSS Inc., Chicago, IL, USA).

\section{RESULTS}

\section{Population characteristics}

The demographic and clinical characteristics of the liver cirrhosis patients and the controls are presented in Table 1. When comparing liver cirrhosis patients with controls, no significant differences were found in terms of age and gender $(P>0.05)$. Moreover, patients with liver cirrhosis were more likely to be infected with chronic hepatitis $\mathrm{B}$ and have a habit of alcohol consumption.

The genotype distributions of IL10 rs1800896 and rs1800872 in the controls were in-line with HWE, and the $P$ values for $I L 10$ rs 1800896 and rs 1800872 were 0.96 and 0.27 , respectively (Table 2). Using the chi-squared test, we found a significant difference in the genotype distributions 
of IL10 rs1800896 between liver cirrhosis patients and controls $\left(\chi^{2}=6.39, \mathrm{P}\right.$ value $\left.=0.04\right)$. By multivariate logistic regression analysis, we found that individuals with the AA genotype of IL10 rs1800896 were associated with an increased risk of liver cirrhosis when compared with the GG genotype in the codominant model $(\mathrm{OR}=2.01,95 \% \mathrm{Cl}=1.10-3.65)$. In the dominant and recessive models, we found that the IL10 rs1800896 polymorphism was correlated with the development of liver cirrhosis (for the dominant model, $\mathrm{OR}=1.46,95 \% \mathrm{Cl}=1.01-2.13$; for the recessive model, $\mathrm{OR}=1.72$, $95 \% \mathrm{Cl}=1.01-3.02)$. However, no significant association was found between the IL10 rs 1800872 polymorphism and the development of liver cirrhosis in codominant, dominant, or recessive models.

\begin{tabular}{|c|c|c|c|c|c|c|}
\hline Variables & Patients $(\mathrm{N}=241)$ & $\%$ & Controls $(\mathrm{N}=254)$ & $\%$ & Chi-squared test & $\mathrm{P}$ value \\
\hline \multicolumn{7}{|l|}{ Age (years) } \\
\hline$<50$ & 110 & 45.64 & 124 & 48.82 & & \\
\hline$\geq 50$ & 131 & 54.36 & 130 & 51.18 & 0.5 & 0.48 \\
\hline \multicolumn{7}{|l|}{ Gender } \\
\hline Females & 84 & 34.85 & 77 & 30.31 & & \\
\hline Males & 157 & 65.15 & 177 & 69.69 & 1.16 & 0.28 \\
\hline \multicolumn{7}{|c|}{ Chronic hepatitis B } \\
\hline No & 104 & 43.15 & 235 & 92.52 & & \\
\hline Yes & 137 & 56.85 & 19 & 7.48 & 139.63 & $<0.001$ \\
\hline \multicolumn{7}{|c|}{ Alcohol consumption } \\
\hline Non-drinkers & 90 & 37.34 & 194 & 76.38 & & \\
\hline Drinkers & 151 & 62.66 & 60 & 23.62 & 77.04 & $<0.001$ \\
\hline \multicolumn{7}{|c|}{ Child-Pugh score } \\
\hline $\mathrm{A}$ & 112 & 46.47 & & & & \\
\hline $\mathrm{B}$ & 93 & 38.59 & & & & \\
\hline $\mathrm{C}$ & 36 & 14.94 & & & & \\
\hline
\end{tabular}

We carried out stratification analysis between the IL10 rs1800896 polymorphism and chronic hepatitis B infection in the risk of liver cirrhosis (Table 3). However, no significant interaction was found between the IL10 rs1800896 polymorphism and chronic hepatitis B infection in the risk of liver cirrhosis.

\begin{tabular}{|c|c|c|c|c|c|c|c|}
\hline SNPs & Patients $(\mathrm{N}=241)$ & $\%$ & Controls $(\mathrm{N}=254)$ & $\%$ & HWE & OR $(95 \% \mathrm{Cl})$ & $P$ value \\
\hline \multicolumn{8}{|c|}{ rs1800896 } \\
\hline \multicolumn{8}{|c|}{ Codominant } \\
\hline GG & 88 & 36.51 & 116 & 45.67 & & 1.0 (Ref.) & - \\
\hline GA & 112 & 46.47 & 111 & 43.70 & & $1.33(0.89-1.98)$ & 0.14 \\
\hline AA & 41 & 17.01 & 27 & 10.63 & 0.96 & $2.01(1.10-3.65)$ & 0.01 \\
\hline \multicolumn{8}{|c|}{ Dominant } \\
\hline GG & 88 & 36.51 & 116 & 45.67 & & 1.0 (Ref.) & - \\
\hline $\mathrm{GA}+\mathrm{AA}$ & 153 & 63.49 & 138 & 54.33 & & $1.46(1.01-2.13)$ & 0.04 \\
\hline \multicolumn{8}{|c|}{ Recessive } \\
\hline $\mathrm{GG}+\mathrm{GA}$ & 200 & 82.99 & 227 & 89.37 & & 1.0 (Ref.) & - \\
\hline $\mathrm{AA}$ & 41 & 17.01 & 27 & 10.63 & & $1.72(1.01-3.02)$ & 0.04 \\
\hline \multicolumn{8}{|c|}{ rs1800872 } \\
\hline \multicolumn{8}{|c|}{ Codominant } \\
\hline AA & 88 & 36.51 & 100 & 39.37 & & 1.0 (Ref.) & - \\
\hline $\mathrm{AC}$ & 104 & 43.15 & 112 & 44.09 & & $1.06(0.70-1.59)$ & 0.79 \\
\hline $\mathrm{CC}$ & 49 & 20.33 & 42 & 16.54 & 0.27 & $1.33(0.78-2.26)$ & 0.27 \\
\hline \multicolumn{8}{|c|}{ Dominant } \\
\hline AA & 88 & 36.51 & 100 & 39.37 & & 1.0 (Ref.) & - \\
\hline $\mathrm{AC}+\mathrm{CC}$ & 153 & 63.49 & 154 & 60.63 & & $1.13(0.77-1.65)$ & 0.51 \\
\hline \multicolumn{8}{|c|}{ Recessive } \\
\hline $\mathrm{AA}+\mathrm{AC}$ & 192 & 79.67 & 212 & 83.46 & & 1.0 (Ref.) & - \\
\hline $\mathrm{CC}$ & 53 & 20.33 & 55 & 16.54 & & $1.06(0.68-1.66)$ & 0.77 \\
\hline
\end{tabular}

SNP = single nucleotide polymorphism; HWE = Hardy-Weinberg equilibrium. 
Table 3. Association between the IL10 rs1800896 polymorphism and chronic hepatitis B infection in the risk of liver cirrhosis.

\begin{tabular}{l|c|c|c|c|c|c}
\hline Variables & \multicolumn{2}{|c|}{ GG } & \multicolumn{2}{c|}{ GA+AA } & OR (95\%Cl) & P value \\
\hline & Patients & Controls & Patients & Controls & & \\
\hline Chronic hepatitis B & 88 & 116 & 153 & 138 & & \\
\hline No & 36 & 105 & 68 & 130 & $1.53(0.92-2.54)$ & 0.08 \\
\hline Yes & 52 & 11 & 85 & 8 & $2.25(0.76-6.86)$ & 0.1 \\
\hline
\end{tabular}

\section{DISCUSSION}

The authors of a previous study have reported that inflammation and the cytokines related to it play a key role in activating stellate cells and causing liver fibrosis, and the inflammatory responses of immune cells and the resultant cytokine expression in the liver may contribute to susceptibility to liver cirrhosis (Yang et al., 2014). In this study, we indicated that the IL10 rs 1800896 polymorphism is associated with the development of liver cirrhosis.

It is reported that the $I L 10$ gene polymorphisms are correlated with the long-term infection of chronic hepatitis B, and thus could influence hepatitis B-related diseases (Gao et al., 2009, 2011; Saxena et al., 2014; Zhang et al., 2014). Gao et al. (2009) studied the relationship between the polymorphisms IL10-1082/-592 and the outcome of hepatitis B virus and hepatitis C virus infection, and found that those polymorphisms influenced persistent hepatitis B virus (HBV) and hepatitis C virus (HCV) infection, clinical outcome, and liver damage. Gao et al. (2011) assessed the relationship between the IL10 gene polymorphisms rs1800896 and rs1800872 and chronic HBV and HCV infection, and found that IL10 rs1800896 appears to affect chronic infection of HBV and HCV replication. Saxena et al. (2014) conducted a case-control study in an Indian population, and found that IL10 rs1800871 and rs1800872 polymorphisms have a strong association with HBV infection-mediated disease progression. Zhang et al. (2014) suggested that the IL10 rs3024490 polymorphism was associated with susceptibility to chronic hepatitis B in a Chinese population. However, we found that the $I L 10$ rs 1800896 polymorphism had no association with chronic hepatitis $B$ in the development of liver cirrhosis. The discrepancies between these results may be caused by differences in ethnicities, study design, and sample size.

Several research teams have reported an association between the IL10 gene polymorphism and liver cirrhosis (Ramezani et al., 2012; Jin et al., 2014; Guo et al., 2015; Liu et al., 2015). Ramezani et al. (2012) suggested that the IL10-592 polymorphism is associated with the outcomes of HBV infection, such as chronic hepatitis, occult HBV infection, liver cirrhosis, or hepatocellular carcinoma. Jin et al. (2014) found that the IL10-592 polymorphism increases the risk of liver cirrhosis, and has an association with chronic hepatitis B infection in the Chinese population. Liu et al. (2015) also conducted a study in a Chinese population, and found that the IL10-1082 polymorphism was correlated with increased risk of liver cirrhosis. Liu et al. (2015) reported that IL-10-592 was associated with the development of HCV-related liver cirrhosis in a Japanese population. In our study, we found that the IL10 rs1800896 polymorphism was associated with the development of liver cirrhosis, but further studies with a large sample size are needed to confirm our results.

In summary, our study suggests that the IL10 rs1800896 polymorphism is associated with the development of liver cirrhosis, but future studies with a larger sample size are needed to assess the role of $I L 10$ polymorphisms in the development of liver cirrhosis. 


\section{Conflicts of interest}

The authors declare no conflict of interest.

\section{REFERENCES}

Ansari N, Makvandi M and Samarbaf-Zadeh AR (2015). Hepatitis B virus Genotyping Among Patients With Cirrhosis. Jundishapur J. Microbiol. 8: e14571. http://dx.doi.org/10.5812/jim.14571

Falasca K, Ucciferri C, Dalessandro M, Zingariello P, et al. (2006). Cytokine patterns correlate with liver damage in patients with chronic hepatitis B and C. Ann. Clin. Lab. Sci. 36: 144-150.

Gao QJ, Liu DW, Zhang SY, Jia M, et al. (2009). Polymorphisms of some cytokines and chronic hepatitis B and C virus infection. World J. Gastroenterol. 15: 5610-5619. http://dx.doi.org/10.3748/wig.15.5610

Gao Q, Zhang S, Wu L, Jia M, et al. (2011). [Association of IL-10-1082 and IL-10-592 polymorphisms with chronic hepatitis $\mathrm{B}$ and/or hepatitis $\mathrm{C}$ virus infection among plasma donors in a rural area of Hebei Province, China]. Wei Sheng Yan Jiu 40: 709-713.

Guo PF, Jin J and Sun X (2015). Influence of IL10 gene polymorphisms on the severity of liver fibrosis and susceptibility to liver cirrhosis in HBV/HCV-infected patients. Infect. Genet. Evol. 30: 89-95. http://dx.doi.org/10.1016/j.meegid.2014.12.011

Hsiang JC, Bai WW, Raos Z, Stableforth W, et al. (2015). Epidemiology, disease burden and outcomes of cirrhosis in a large secondary care hospital in South Auckland, New Zealand. Intern. Med. J. 45: 160-169. http://dx.doi.org/10.1111/ imj.12624

Jin XY, Wang YQ, Yan T, Wang J, et al. (2014). Interleukin-10 gene promoter polymorphism and susceptibility to liver cirrhosis. Hepatogastroenterology 61: 442-446.

Lin CW, Lin CC, Mo LR, Chang CY, et al. (2013). Heavy alcohol consumption increases the incidence of hepatocellular carcinoma in hepatitis B virus-related cirrhosis. J. Hepatol. 58: 730-735. http://dx.doi.org/10.1016/j.jhep.2012.11.045

Liu Y, Yu MC, Zhang AQ, Wang YB, et al. (2015). Interleukin-10 gene promoter polymorphism and risk of liver cirrhosis. Genet. Mol. Res. 14: 1229-1234. http://dx.doi.org/10.4238/2015.February.13.1

Nelson DR, Lauwers GY, Lau JY and Davis GL (2000). Interleukin 10 treatment reduces fibrosis in patients with chronic hepatitis C: a pilot trial of interferon nonresponders. Gastroenterology 118: 655-660. http://dx.doi.org/10.1016/S0016$\underline{5085(00) 70134-X}$

Ramezani A, Banifazl M, Mamishi S, Sofian M, et al. (2012). The influence of human leukocyte antigen and IL-10 gene polymorphisms on hepatitis B virus outcome. Hepat. Mon. 12: 320-325. http://dx.doi.org/10.5812/hepatmon.6094

Saxena R, Chawla YK, Verma I and Kaur J (2014). Association of interleukin-10 with hepatitis B virus (HBV) mediated disease progression in Indian population. Indian J. Med. Res. 139: 737-745.

Tsukamoto H (1998). Is interleukin-10 antifibrogenic in chronic liver injury? Hepatology 28: 1707-1709. http://dx.doi.org/10.1002/ hep. 510280635

Yang AM, Wen LL, Yang CS, Wang SC, et al. (2014). Interleukin 10 promoter haplotype is associated with alcoholic liver cirrhosis in Taiwanese patients. Kaohsiung J. Med. Sci. 30: 291-298. http://dx.doi.org/10.1016/j.kjms.2014.02.016

Zhang TC, Zhang WF, Zhao YQ, Pan FM, et al. (2014). Gene variation in IL10 and susceptibility to chronic hepatitis B. J. Infect. 69: 75-80. http://dx.doi.org/10.1016/j.jinf.2014.03.003 